\title{
Future Contributions to Journal of Statistical Physics
}

Functional Integral Approach to Classical Statistical Dynamics Roderick V. Jensen

Exact Fixed Points in Discrete Hydrodynamics

P. B. Visscher

Molecular Dynamics Simulations of One-Dimensional Lennard-Jones Systems

Marvin Bishop, Marie Derosa, and Joanne Lalli

Central Limit Theorems for Percolation Models

$J$. Theodore Cox and Geoffrey Grimmett

Deviation of $1 / f$ Voltage Fluctuations from Scale-Similar Gaussian Behavior

Mark Nelkin and A.-M. S. Tremblay

Analytic and Numerical Evidence from Quantum Field Theory for the Hyperscaling Relation $d \nu=2 \Delta-\gamma$ in the $d=3$ Ising Model R. Schrader and E. Tränkle

The Central Limit Theorem for the Spectrum of the Sional Schrödinger Operator

A. Ja. Režnikova

Mean Field Bounds on the Magnetization for Ferromagnetic Spin Models Paul A. Pearce

The Incoherent Scattering Function and Related Correlation Functions in Hard Sphere Fluids at Short Times

I. M. de Schepper amd M. H. Ernst

Field Theory of the Two-Dimensional Ising Model: II. Nonlocal Specific Heat

Richard A. Ferrell 